\title{
An Autologous Anti-Inflammatory Protein Solution Yielded a Favorable Safety Profile and Significant Pain Relief in an Open-Label Pilot Study of Patients with Osteoarthritis
}

\author{
Jason Hix, ${ }^{1}$ Mark Klaassen, ${ }^{1}$ Ryan Foreman, ${ }^{1}$ Edith Cullen, ${ }^{1}$ Krista Toler, ${ }^{2}$ William King, ${ }^{2, *}$ and Jennifer Woodell-May ${ }^{2}$
}

\begin{abstract}
Osteoarthritis $(\mathrm{OA})$ is a progressive and degenerative disease, which may result in significant pain and decreased quality of life. Recent updates in our understanding of OA have demonstrated that it is a whole joint disease that has many similarities to an unhealed wound containing inflammatory cytokines. The nSTRIDE Autologous Protein Solution (APS) Kit is a medical device under development for the treatment of OA. The APS Kit processes a patient's own blood at the point of care to contain high concentrations of anti-inflammatory cytokines and anabolic growth factors. This study assessed the safety and treatment effects of a single intra-articular injection of APS. Eleven patients were enrolled in this study. Sufficient blood could not be drawn from one patient who was subsequently withdrawn, leaving 10 patients treated. Minor adverse events (AEs) were experienced by seven subjects (63.6\%). There was one serious AE (diverticulitis) unrelated to the device or procedure. One subject experienced AEs that were judged "likely" to be procedure related (arthralgia/musculoskeletal discomfort) and all resolved within 6 days of injection. All other AEs were unrelated to the device or procedure. Western Ontario and McMaster Universities Osteoarthritis Index (WOMAC) pain scores improved significantly over time (ANOVA, $p<0.0001,12.0 \pm 1.2$ preinjection, $3.3 \pm 2.9$ one year postinjection, and $72.5 \%$ WOMAC pain improvement). There was significant positive correlation between white blood cell concentration in APS and improvement in WOMAC pain scores.
\end{abstract}

Keywords: AAl; APS; IL-1ra; intra-articular; OA

\section{Introduction}

Osteoarthritis (OA) is a debilitating disease that is one of the leading causes of pain and disability worldwide. ${ }^{1}$ $\mathrm{OA}$ is considered a whole-organ disease of the joint, ${ }^{2}$ as an inflammatory disease, ${ }^{3}$ but with chronic low-grade inflammation, ${ }^{4}$ in which there is an association between biomarkers of tissue inflammation and progression of $\mathrm{OA},{ }^{5}$ synovitis in which chondrocytes and macrophages overproduce inflammatory mediators such as IL-1 $\beta$ and TNF, ${ }^{6,7}$ increased expression of IL- $1 \beta$ gene in peripheral blood mononuclear cell leukocyte of patients with symptomatic knee $\mathrm{OA}$ and radiographic progression of $\mathrm{OA},{ }^{8}$ and sex-specific association between painful joint burden in $\mathrm{OA}$ and systemic inflammation. ${ }^{9}$
There have been many attempts to modify or slow the progression of OA with limited success. ${ }^{10}$ Emerging concepts of OA disease progression may instruct the development of new therapies. Recent research has suggested that $\mathrm{OA}$ is a whole-joint disease ${ }^{11}$ that is analogous to a nonhealing wound. ${ }^{12} \mathrm{OA}$ affects not only cartilage but also the synovial tissue and subchondral bone. ${ }^{11}$ In this model, an event triggers the production of inflammatory cytokines, which induce cells in the joint to secrete proteases and glycosaminoglycan-degrading enzymes. ${ }^{13}$ The resulting extracellular tissue fragments bind to cells that induce the production of even more inflammatory cytokines. ${ }^{3}$ The body attempts to heal this "wound" by cellular auto-debridement ${ }^{12}$ and increases in the 
number of M2-prohealing macrophages, ${ }^{14}$ but in adult humans, the healing response is insufficient to stop OA.

Successful wound healing in humans follows a standard pathway and requires the presence of both antiinflammatory cytokines and tissue healing molecules. In early wound healing, platelets degranulate, releasing anabolic growth factors that recruit white blood cells (WBCs) ${ }^{15}$ WBCs are the primary source of interleukin1 receptor antagonist (IL-1ra), ${ }^{16}$ a key inhibitor of inflammatory IL-1, and soluble tumor necrosis factor receptors (sTNF-R), which block inflammatory TNF $\alpha .{ }^{17}$ An autologous anti-inflammatory (AAI) therapy that could provide pro-tissue healing molecules and multiple inhibitors of inflammatory cytokines could potentially heal the unresolved wound, which is OA, thus restoring homeostasis to the joint and providing long-term pain relief.

Research has demonstrated that the Autologous Protein Solution (APS) Kit produces an AAI output, APS, at the point of care from a small amount of patient's own blood. In vitro experiments have shown that APS inhibits production of proteases by chondrocytes ${ }^{17}$ and inflammatory cytokines from macrophages in inflammatory cell culture environments. ${ }^{18}$ APS protected collagen and glycosaminoglycan in cartilage explants when cultured with inflammatory cytokines and induced a healing response in chondrocytes. ${ }^{19}$ Furthermore, APS has been shown to induce M2 "pro-healing" macrophage polarization in cell culture. ${ }^{20}$ This reparative response was also demonstrated in a randomized and controlled rat medial meniscal tear model, in which APS significantly decreased cartilage degradation and improved the total joint score compared to saline controls. ${ }^{21}$ In randomized and controlled large animal studies, APS has improved lameness in both horses ${ }^{22}$ and dogs with naturally occurring $\mathrm{OA} .{ }^{23}$ A human clinical study demonstrated that APS contained high concentrations of anti-inflammatory cytokines and anabolic growth factors from 105 patients with OA. ${ }^{24}$ Finally, a feasibility study and a randomized, controlled study have demonstrated that the output of the APS device is safe when injected intra-articularly and provided significantly improved pain compared to saline control 1 year after a single injection of APS. ${ }^{25-27}$ Together, these cell culture, explant, animal, and human studies suggest that APS has properties that could potentially resolve the unhealed "wound," which is OA, restore homeostasis to the joint, and provide long-term pain relief for patients with OA.

The purpose of this open-label, single-center, nonrandomized, prospective safety evaluation was to further assess the safety of a single injection of APS in patients with painful unilateral knee OA. The primary end-point of this study was to characterize the safety profile of APS subsequent to the 1 month follow-up. Secondary safety end-points included careful monitoring of the index knee at $15 \mathrm{~min}, 1 \mathrm{~h}$, and $2 \mathrm{~h}$ postinjection and measures of clinical efficacy, including Western Ontario and McMaster Universities Osteoarthritis Index (WOMAC), Knee Injury and Osteoarthritis Outcome Score (KOOS), Numeric Rating Scale (NRS) pain, and global assessments. The Outcome Measures in Rheumatology-Osteoarthritis Research Society International (OMERACT-OARSI) set of responder criteria was utilized to determine the number of treatment responders. ${ }^{28}$ The incidence of patients taking acetaminophen/paracetamol for OA pain was tracked as well. MRI assessment of the joint tissues provided a comparison of structural changes from baseline to 12 months. X-ray assessments were also reviewed for structural changes from baseline to 12 months.

\section{Materials and Methods}

Study design

This was a single-center, single-arm, nonrandomized, prospective safety evaluation of a single APS injection. A total of 11 patients were enrolled, of which 10 patients received an injection prepared using the nSTRIDE APS Kit (Zimmer Biomet, Warsaw, IN). The device was used under an Investigational Device Exemption (IDE 15978). Sufficient blood could not be drawn from one subject for device processing. All subjects met the specific inclusion and exclusion criteria (Supplementary Tables S1 and S2). The included population could generally be characterized as patients with painful unilateral knee OA, who had not been able to get satisfactory pain relief with other treatments.

Patients provided written informed consent and underwent screening assessments, including demographics, medical history, physical examination, knee exam, knee radiograph, a urine pregnancy test (if applicable), WOMAC LK 3.1, and medication usage. MRI and Xray imaging were performed before the injection procedure. Upon confirmation of eligibility, subjects were scheduled for the treatment visit.

Subjects returned to the clinic within 4 weeks of screening for the injection visit. Eligibility was confirmed by WOMAC LK 3.1 and a urine pregnancy test (when applicable). Before undergoing treatment, baseline evaluations were performed including knee examination, Global Impression of Severity scales, KOOS, and NRS. 
All subjects had three blood samples drawn: one $60 \mathrm{~mL}$ volume $(55 \mathrm{~mL}$ blood $+5 \mathrm{~mL}$ Anticoagulant Citrate Dextrose Solution-Formula A [ACD-A]) was processed using the APS Kit and used for treatment. A second (processed) $60 \mathrm{~mL}$ volume was used for analytical testing and one whole blood sample $(11 \mathrm{~mL}$ blood $+1 \mathrm{~mL}$ ACD-A) was used for analytical testing. After any available joint fluid was aspirated, APS was injected into the joint. Subjects were monitored in the clinic for $2 \mathrm{~h}$ after the injection, and a knee/injection site examination was completed at $15 \mathrm{~min}, 1 \mathrm{~h}$, and $2 \mathrm{~h}$ postinjection.

Before discharge, subjects were instructed not to exceed their preinjection physical activity level for 14 days postinjection. Participants were also instructed to contact their physician's office if they intended to substantially increase their activity levels while they were study subjects. Each subject was given acetaminophen and a diary to record all acetaminophen use and the reason for that use. Subjects were advised to abstain from acetaminophen for at least $48 \mathrm{~h}$ before follow-up visits.

Safety was assessed in a follow-up phone call 1 day after treatment and safety and efficacy measures were assessed at postinjection visits at week 1 and months 1 , 3,6 , and 12. All safety assessments that were used in this study are commonly used standard measurements. In addition to adverse events (AEs), safety assessments, including knee examination and injection site reactions occurring during or after the procedure, were reported. Any and all AEs that occurred until the 12-month followup were recorded. All AEs reported to or identified by investigative center personnel were documented. AEs were summarized, including documentation of the onset and resolution date(s) (if available), as well as management and outcome. Assessments of severity, seriousness, relatedness, and whether they were anticipated were recorded.

The following assessments or information were collected at all follow-up visits: WOMAC LK 3.1; knee exam; acetaminophen use and reconciliation; Global Impression of Severity Scales by subject and investigator; concomitant medication use; KOOS; NRS knee pain scale, and AEs. At 12 months, a standing radiograph of the index knee was done and at 3 and 12 months, a knee MRI was done, for the evaluation of structural changes in the joint. The OMERACTOARSI set of responder criteria were calculated as previously described (Supplementary Fig. S1). ${ }^{28}$

\section{Investigational device description}

The nSTRIDE $^{\circledR}$ APS Kit (Zimmer Biomet, Warsaw, IN) is a sterile single-use unit containing two blood processing devices with a vial of ACD-A. The first device is the Cell Separator that processed $55 \mathrm{~mL}$ of blood and $5 \mathrm{~mL}$ of ACD-A to produce a cell solution. The second device is the Concentrator device that contains polyacrylamide beads to further concentrate the cell solution and produce an injectable output, the APS.

\section{Characterization of APS and whole blood}

APS and whole blood samples were transported to Zimmer Biomet Biologics for characterization. Complete blood count analysis was conducted on a Cell Dyn Sapphire (Abbott, Abbott Park, IL). Remaining samples were frozen at $-80^{\circ} \mathrm{C}$ until ELISA analysis (R\&D Systems, Minneapolis, MN).

\section{Imaging analysis}

Knee X-rays suitable for determining a KellgrenLawrence (K-L) grade for the index knee were taken during the screening process, unless an X-ray of adequate quality was available and was taken within 6 months of the injection. A second X-ray was taken at 12 months postprocedure. In both instances, a K-L grade was determined. MRI images were obtained preinjection and again at 3 and 12 months postprocedure. All MRI images were evaluated by a central laboratory according to the MRI Osteoarthritis Knee Score criteria.

\section{Statistical analysis methods}

The evaluable population included all patients who received an injection of APS. No missing data were imputed. Analyses included all data regardless of whether collected inside the protocol-defined window. AEs were standardized using the Medical Dictionary for Regulatory Activities. Characterization included a summary of all AEs and descriptive statistics, including, but not limited to, $\mathrm{AE}$ incidence overall and per patient, $\mathrm{AE}$ type, $\mathrm{AE}$ severity, AE relatedness, AE duration, $\mathrm{AE}$ onset, and the proportion of patients with one or more AEs.

Subject- and clinician-reported outcome measuresWOMAC LK 3.1, Global Impression-Severity Scales, KOOS, and NRS knee pain-were summarized and thoroughly characterized using descriptive statistics, including error estimates. Statistics included mean, median, range, minimum, maximum, frequency, cumulative frequency percent, and cumulative percent. A repeated measure ANOVA and/or a Wilcoxon signed-rank test were completed for each outcome measure. If a significant trend was identified at $\alpha \leq 0.05$ in a planned test, then statistical significance between time points was explored further using pair-wise comparison. 


\section{Results}

The target population for this study was patients diagnosed with painful unilateral knee OA, who were not able to get satisfactory pain relief with prior treatments. After confirmation of all eligibility criteria, the patients were enrolled and followed up for 12 months. A total of 11 patients were enrolled at one center, of which 10 patients were treated and completed the 12-month follow-up. One patient was withdrawn before treatment due to inadequate blood draw. The average age of the subjects in this study was $58.8 \pm 9.5$ years (average \pm standard deviation) (43-73 years old). In this study, seven subjects were male and three subjects were female. The average BMI was $29.0 \pm 3.9 \mathrm{~kg} / \mathrm{m}^{2}$ $\left(21.3-35.1 \mathrm{~kg} / \mathrm{m}^{2}\right)$.

The average APS volume was $2.9 \pm 0.2 \mathrm{~mL}$. The average APS WBC concentration was $49.5 \pm 12.5 \mathrm{k} / \mu \mathrm{L}(11.0 \times$ baseline) and the platelet concentration was $377.8 \pm$ $102.4 \mathrm{k} / \mu \mathrm{L}(3.0 \times)$. The APS contained high concentrations of anti-inflammatory cytokines and low concentrations of inflammatory cytokines (Table 1). The average amount of IL-1ra in APS was 179.9 $\pm 63.1 \mathrm{ng}$ (Supplementary Table S3). The IL-1ra:IL-1 $\beta$ ratio was $7960 \pm$ 3637 (IL-1 $\mathrm{ra}_{\mathrm{pg} / \mathrm{mL}} / \mathrm{IL}-1 \beta_{\mathrm{pg} / \mathrm{mL}}$ ) (Supplementary Table S4).

As this was a safety study, all AEs were recorded even if they were not related to the device or procedure. No unanticipated AE occurred during the study that was attributable to the procedure or the device. Four AEs were determined "Likely" to be related to the procedure by the investigator. These were three incidents of arthralgia and one incident of musculoskeletal discomfort, all reported by one subject. All were judged to be "Mild," and all resolved within 6 days following the injection procedure. No $\mathrm{AE}$ was determined by the investigator to be related to the device. One serious AE occurred (diverticulitis), and this event was determined to be unrelated to either the procedure or the device. In addition, there were no noteworthy or unanticipated AEs observed during the knee examinations. Minor AEs were experienced by seven subjects (63.6\%) (Table 2).
WOMAC pain scores improved significantly over time (ANOVA, $p<0.0001),(12.0 \pm 1.2)$ before injection and $(3.3 \pm 2.9) 1$ year postinjection. This corresponded to a $72.5 \%$ WOMAC pain improvement on average. WOMAC stiffness $(p \leq 0.0371)$, WOMAC function $(p \leq 0.0064)$, and WOMAC total score $(p \leq 0.0064)$ were also improved at all time intervals compared to baseline. The mean KOOS pain score before injection was $36.9 \pm 16.2$. One week after the injection, the mean KOOS pain score was $56.7 \pm 16.5$, and it was $79.7 \pm 16.2$ one year after the injection. Preinjection KOOS pain was significantly different from KOOS pain at all postinjection intervals $(p \leq 0.0029)$. Also, the KOOS symptom $(p \leq 0.0269)$, KOOS stiffness $(p \leq 0.0420)$, KOOS function $(p \leq 0.0050)$, and KOOS sport function $(p \leq 0.0231)$ were significantly improved at all postinjection intervals. Repeated measures ANOVA was conducted to determine whether a difference between intervals existed for NRS pain across time intervals $(p<0.0001)$. NRS pain at baseline was 5.9 \pm 1.9 . The lowest level of mean pain was observed at 1 year postprocedure $(1.6 \pm 1.6)$, and pain trended toward improvement consistently from interval to interval as time progressed. When compared to preinjection, each time interval was considered significantly different $(p \leq 0.0065)$ (Fig. 1).

For patient-reported Global Impression, the $p$-value associated with a repeated means ANOVA to determine whether a difference between intervals exists was $p=0.0002$. At baseline, the most frequently reported score was severe $(n=4)$, followed by moderate $(n=3)$ and mild $(n=3)$. One week after the injection, mild, moderate, and marked were each scored in three cases, severe in one case. At 1 year following treatment, two cases were scored as normal, two were borderline, four were mild, and two were moderate (Fig. 2).

Four subjects used analgesics for study knee pain postinjection. All of these subjects were taking analgesics for pain in their study knee before the injection procedure. In all cases except one, acetaminophen

Table 1. Cytokine Concentrations $(\mathrm{pg} / \mathrm{mL})$ in Autologous Protein Solution

\begin{tabular}{|c|c|c|c|c|c|}
\hline & IL-1ra (pg/mL) & $\mathrm{IL}-1 \beta(\mathrm{pg} / \mathrm{mL})$ & sIL-1RII (pg/mL) & $\operatorname{TNF} \alpha(\mathrm{pg} / \mathrm{mL})$ & sTNF-RII (pg/mL) \\
\hline Average & $63,739.6$ & 16.5 & $26,216.8$ & BR & 6347.7 \\
\hline Standard deviation & $23,556.3$ & 25.5 & 6126.1 & BR & 1425.2 \\
\hline Maximum & $120,783.1$ & 88.0 & $35,135.5$ & BR & 9458.9 \\
\hline Minimum & $38,335.8$ & $\mathrm{BR}$ & $12,427.6$ & BR & 4670.9 \\
\hline
\end{tabular}

$\mathrm{BR}$, below the range of the ELISA assay; IL-1ra, interleukin-1 receptor antagonist; sTNF-R, soluble tumor necrosis factor receptor. 
Table 2. Adverse Event Summary

\begin{tabular}{lc}
\hline Total No. of AEs & $\mathbf{2 7}$ \\
\hline No. of patients with at least one AE & 7 \\
No. of device-related AEs & 0 \\
No. of severe AEs & 1 \\
No. of serious AEs & 1
\end{tabular}

AEs, adverse events.

was taken for study knee pain. In one patient, Ibuprofen was used at 1 week postprocedure, and then discontinued. In addition, one patient used multiple analgesics at 3 months postprocedure for diverticulitis and subsequently discontinued analgesic use. Reported analgesic use did not account for pain improvement observed in this study.

There were variable responses observed on imaging. A larger study would be likely required to identify any clear morphological improvements. There was no observed worsening attributable to the APS injection. The number of OMERACT-OARSI responders increased from (5/10) 1 week postinjection to $(10 / 10) 1$ year postinjection (Table 3 ).
APS WBC concentration was positively correlated with WOMAC pain improvement 1 year post-treatment ( $p=0.038)$ (Fig. 3).

\section{Discussion}

A single injection of APS prepared using the APS Kit yielded a safety profile that compares favorably to other intra-articular therapies. ${ }^{29}$ The APS contained high concentrations of WBCs and therefore contained high concentrations of anti-inflammatory cytokines, although low concentrations of inflammatory cytokines. In this study, the APS Kit had a favorable safety profile with no AEs related to the device. Subjects had a mean 72.5\% WOMAC pain improvement 1 year postinjection and $100 \%$ of the subjects were OMERACT-OARSI responders. Also, the concentration of WBCs in APS significantly correlated with improved WOMAC pain improvement. These results provide further evidence that the intra-articular injection of APS is safe. Furthermore, these results demonstrate the reproducibility of previous clinical studies of this device. ${ }^{26}$

The safety profile and device performance of a single intra-articular injection of APS in this study was
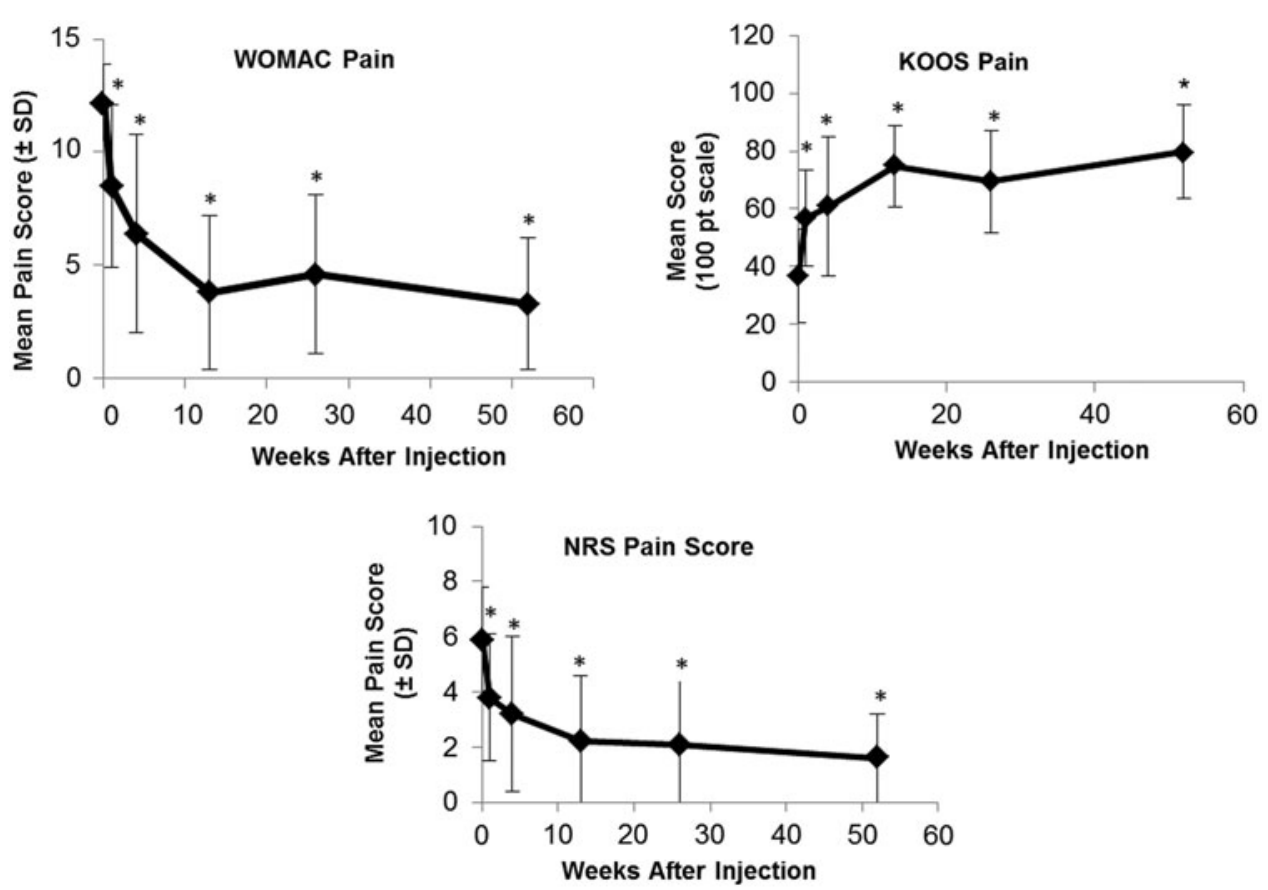

FIG. 1. WOMAC pain scores, KOOS pain scores, and NRS pain scores $(n=10)$. *Indicates significantly different from baseline $(p<0.05)$. KOOS, Knee Injury and Osteoarthritis Outcome Score; NRS, Numeric Rating Scale; WOMAC, Western Ontario and McMaster Universities Osteoarthritis Index. 


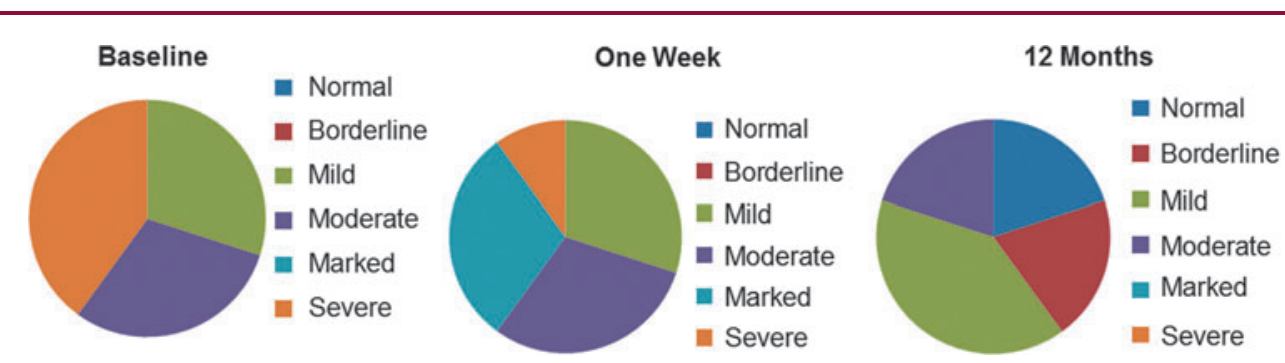

FIG. 2. Patient-reported Global Impression scores at baseline, 1 week postinjection, and 12 months postinjection.

similar to previous studies. In this study, there were no unanticipated AEs that were attributable to the procedure or the device. Similarly, in a previous 11-patient single-arm feasibility study, there were no AEs that were reported by the investigator as related to the device. ${ }^{30}$ In this study and in the previous safety study, all AEs resolved quickly without further intervention. The APS Kit produced an output with high concentrations of anti-inflammatory cytokines, including IL-1 ra, in this study $(63,740 \pm 23,556 \mathrm{pg} / \mathrm{mL})$ and in the previous feasibility study $(57,511 \pm 24,272 \mathrm{pg} / \mathrm{mL}){ }^{31}$ The high production of anti-inflammatory cytokines in both studies can be contributed to the high concentration of WBCs $(49.5 \pm 12.5 \mathrm{k} / \mu \mathrm{L}$ in this study and $49.4 \pm 15.3 \mathrm{k} / \mu \mathrm{L}$ in the previous study). ${ }^{31}$ The high concentrations of anti-inflammatory cytokines and WBCs may have helped modify the "wound," which is OA, and could have contributed to the positive secondary efficacy outcomes in this study.

The secondary efficacy outcomes were also very similar between studies. In both studies, subjects had significantly improved WOMAC pain scores 1 week postinjection. In this study, subjects had an average 62\% WOMAC pain improvement 6 months postinjection and in the previous study, subjects had an average $74 \%$ WOMAC pain improvement 6 months postinjection. ${ }^{30}$

Table 3. OMERACT-OARSI Responder Status

\begin{tabular}{lcc}
\hline OMERACT-OARSI classification & \\
\hline Interval & Responders & Nonresponders \\
\hline 1 Week & 5 & 5 \\
4 Weeks & 7 & 3 \\
3 Months & 8 & 2 \\
6 Months & 8 & 2 \\
1 Year & 10 & 0 \\
\hline
\end{tabular}

OMERACT-OARSI, Outcome Measures in Rheumatology-Osteoarthritis Research Society International.
In both studies, the concentration of WBCs was correlated with improved WOMAC pain improvement. There previously has been a debate about the role of WBCs in autologous therapy-based intra-articular injections for the treatment of OA, but this study and other pre-clinical and clinical studies indicate that clinical scientific consensus has been met for most active researchers in the field. First, there is now a large body of evidence that in vitro models of OA do not translate well to clinical results relating to improvements in pain. ${ }^{32}$ The only head-to-head study of leukocyte-rich platelet-rich plasma versus leukocytepoor platelet-rich plasma (PRP) did not show any clinical differences, but both PRPs in this study were injected once fresh and then pipetted into aliquots that were frozen, and then thawed for the subsequent injections ${ }^{33}$; APS is different in that it is never frozen. The freezing could compromise the viability of WBCs. ${ }^{34}$ Direct intra-articular measurements of synovial fluid after leukocyte-rich PRP injections have shown no upregulation of inflammatory cytokines. ${ }^{35}$ Indeed, a large case series of bone marrow concentrate

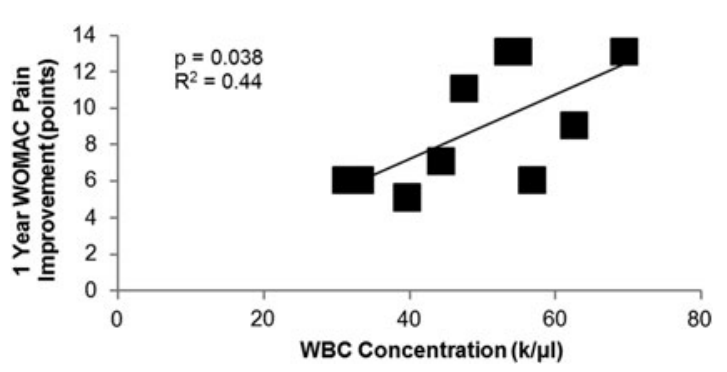

FIG. 3. Correlation analysis of WBC concentrations with improvement in WOMAC pain scores. WBC, white blood cell. 
injections for $\mathrm{OA}$ have demonstrated the safety of high concentrations of WBC in autologous therapies. ${ }^{36}$ Finally, WBC concentration in both this study and the previous feasibility study of this device was significantly correlated with improved WOMAC pain scores. ${ }^{31}$ Together these data support the model of OA as an unhealed "wound" and, as in every other type of wound healing in adult mammals, WBCs play a necessary and positive role.

Future studies of autologous point-of-care therapies addressing patients with painful OA might keep in mind that these patients can present with active synovitis, which is a condition of tissue inflammation and is associated with OA progression. Biomarkers of tissue inflammation such as urinary type II collagen, serum cartilage oligomeric protein, and matrix metalloproteinasedependent degradation of C-reactive protein should be presented. It could be also useful to consider a sex-specific association between painful joint and systemic inflammation. This could allow a more personalized medicine.

\section{Conclusion}

The primary objective of this pilot investigation was to assess the safety of the APS Kit in patients with knee $\mathrm{OA}$. The safety evaluation yielded a positive profile of the subject device. No device-related AEs were reported throughout the clinical study. Furthermore, considering the small number of subjects in this study, the consistency of favorable results among diverse efficacy measurements such as the WOMAC, KOOS, Global Impression, and OMERACT-OARSI responder rate was remarkable. Improvement continued through 12 months postprocedure, indicating the durability of a single APS injection. The results of this clinical investigation indicate that treatment with a single, intraarticular injection of APS in patients with knee OA can be considered safe and warrants further investigation of the nSTRIDE APS Kit.

\section{Acknowledgment}

The authors would like to acknowledge Kelly Ganz for her assistance on this trial.

\section{Author Disclosure Statement}

W.J.K., K.T., and J.W.M. are employees of Zimmer Biomet. M.K. has been a consultant for Zimmer Biomet.

\section{References}

1. Neogi T. The epidemiology and impact of pain in osteoarthritis. Osteoarthritis Cartilage. 2013;21:1145-1153.
2. Loeser RF, Goldring SR, Scanzello CR, et al. Osteoarthritis: a disease of the joint as an organ. Arthritis Rheum. 2012;64:1697-1707.

3. Berenbaum F. Osteoarthritis as an inflammatory disease (osteoarthritis is not osteoarthrosis!). Osteoarthritis Cartilage. 2013;21:16-21.

4. Robinson WH, Lepus CM, Wang Q, et al. Low-grade inflammation as a key mediator of the pathogenesis of osteoarthritis. Nat Rev Rheumatol. 2016; 12:580-592.

5. Hosnijeh FS, Siebuhr AS, Uitterlinden AG, et al. Association between biomarkers of tissue inflammation and progression of osteoarthritis: evidence from the Rotterdam study cohort. Arthritis Res Ther. 2016;18:81.

6. Pelletier JP, Martel-Pelletier J, Abramson SB. Osteoarthritis, an inflammatory disease: potential implication for the selection of new therapeutic targets. Arthritis Rheum. 2001;44:1237-1247.

7. Bondeson J, Blom $A B$, Wainwright $S$, et al. The role of synovial macrophages and macrophage-produced mediators in driving inflammatory and destructive responses in osteoarthritis. Arthritis Rheum. 2010;62:647-657.

8. Attur M, Belitskaya-Lévy I, Oh C, et al. Increased interleukin-1 $\beta$ gene expression in peripheral blood leukocytes is associated with increased pain and predicts risk for progression of symptomatic knee osteoarthritis. Arthritis Rheum. 2011;63:1908-1917.

9. Perruccio A, Chandran V, Power J, et al. Systemic inflammation and painful joint burden in osteoarthritis: a matter of sex? Osteoarthritis Cartilage. 2017;25:53-59.

10. Smelter E, Hochberg MC. New treatments for osteoarthritis. Curr Opin Rheumatol. 2013;25:310-316.

11. Poole AR. Osteoarthritis as a whole joint disease. HSS J. 2012;8:4-6.

12. Kraus VB. Osteoarthritis: The Zinc Link. Nature. 2014;507:441-442.

13. Fernandes JC, Martel-Pelletier J, Pelletier JP. The role of cytokines in osteoarthritis pathophysiology. Biorheology. 2002;39:237-246.

14. Daghestani HN, Pieper CF, Kraus VB. Soluble macrophage biomarkers indicate inflammatory phenotypes in patients with knee osteoarthritis. Arthritis Rheum. 2015;67:956-965.

15. Borysenko M, Beringer T. Functional Histology. Little Brown: Boston, 1984.

16. Jordan M, Otterness IG, Ng R, et al. Neutralization of endogenous IL-6 suppresses induction of IL-1 receptor antagonist. Immunology. 1995;154: 4081-4090.

17. Woodell-May JE, Matuska A, Oyster M, et al. Autologous protein solution inhibits MMP- 13 production by IL- $1 \beta$ and TNF $\alpha$-stimulated human articular chondrocytes. J Orthop Res. 2011;29:1320-1326.

18. O'Shaughnessey KM, Panitch A, Woodell-May JE. Blood-derived antiinflammatory protein solution blocks the effect of IL-1 $\beta$ on human macrophages in vitro. Inflamm Res. 2011;60:929-936.

19. Matuska A, O'Shaughnessey KM, King WJ, et al. Autologous solution protects bovine cartilage explants from IL-1 $\alpha$-and TNF $\alpha$-induced cartilage degradation. Orthop Res. 2013;31:1929-1935.

20. King WJ, Han B, Woodell-May JE. An autologous protein solution induces the M2 pro-healing phenotype of cultured macrophages. In: Regenerative Medicine Workshop. Hilton Head, SC, 2014.

21. King WJ, Bendele AM, Marohl T, et al. Human blood-based antiinflammatory solution inhibits osteoarthritis progression in a meniscaltear rat study. Orthop Res. 2017;35:2260-2268.

22. Bertone AL, Ishihara A, Zekas $L$, et al. Evaluation of a single intra-articular injection of autologous protein solution for treatment of osteoarthritis in horses. Am J Vet Res. 2014;75:141-151.

23. Wanstrath AW, Hettlich BF, Su L, et al. Evaluation of a single intra-articular injection of autologous protein solution for treatment of osteoarthritis in a canine population. Vet Surg. 2016;45:764-774.

24. O'Shaughnessey KM, Matuska A, Hoeppner J, et al. Autologous protein solution prepared from the blood of osteoarthritic patients contains an enhanced profile of anti-inflammatory cytokines and anabolic growth factors. Orthop Res. 2014:1349-1355.

25. King WJ, van der Weegen W, van Drumpt R, et al. Characterizing the Relationship between White Blood Cell and IL-1ra Concentration in Whole Blood and Decreased Osteoarthritis Pain in an Open-Label Study of Autologous Protein Solution. European Federation of National Associations of Orthopaedics \& Traumatology Congress. 16th Annual Meeting, Geneva, 2015.

26. van der Weegen W, van Drumpt $R$, Toler $K$, et al. Safety and outcomes following a single autologous protein solution injection for knee osteoarthritis: A pilot study. Int Cartilage Repair Soc. 2015:6471.

27. Kon E, Engebretsen L, Verdonk PC, et al. Clinical outcomes of an autologous protein solution injection for knee osteoarthritis: a 1-year pilot doubleblinded randomized controlled trial. Am J Sports Med. 2017 (In Press). 
28. Pham T, van der HD, Altman RD, et al. OMERACT-OARSI initiative: Osteoarthritis Research Society International set of responder criteria for osteoarthritis clinical trials revisited. Osteoarthritis Cartilage. 2004;12:389-399.

29. Altman RD, Akermark C, Beaulieu AD, et al. Efficacy and safety of a single intra-articular injection of non-animal stabilized hyaluronic acid (NASHA) in patients with osteoarthritis of the knee. Osteoarthritis Cartilage. 2004; 12:642-649.

30. van Drumpt R, van der Weegen W, King WJ, et al. Safety and treatment effectiveness of a single autologous protein solution injection in patients with knee osteoarthritis. Biores Open Access. 2016;5:261-268.

31. King WJ, van der Weegen W, van Drumpt R, et al. White blood cell concentration correlates with increased concentrations of IL-1 ra and improvement in WOMAC pain scores in an open-label safety study of autologous protein solution. J Exp Orthop. 2016;3:9.

32. Johnson $\mathrm{Cl}$, Argyle DJ, Clements DN. In vitro models for the study of osteoarthritis. Vet J. 2016;209:40-49.

33. Filardo G, Kon E, Pereira Ruiz MT, et al. Platelet-rich plasma intra-articular injections for cartilage degeneration and osteoarthritis: single- versus double-spinning approach. Knee Surg Sports Traumatol Arthrosc. 2012; 20:2082-2091.

34. Huggins CE. A general system for the preservation of blood by freezing Long-term Preservation of Red Blood Cells: A Conference Sponsored by the Committee on Blood and Transfusion Problems, Division of Medical Sciences, National Academy of Science-National Research Council, 21 and 22 May 1964. Proceedings by Mary T. Sproul (ed.): National Academies; p. 160; 1965.

35. Mariani E, Canella V, Cattini L, et al. Leukocyte-rich platelet-rich plasma injections do not up-modulate intra-articular pro-inflammatory cytokines in the osteoarthritic knee. PLoS One. 2016;11:e0156137.

36. Centeno C, Pitts J, Al-Sayegh H, et al. Efficacy of autologous bone marrow concentrate for knee osteoarthritis with and without adipose graft. Biomed Res Int 2014;2014:370621.
Cite this article as: Hix J, Klaassen M, Foreman R, Cullen E, Toler K, King W, Woodell-May J (2017) An autologous anti-inflammatory protein solution yielded a favorable safety profile and significant pain relief in an open-label pilot study of patients with osteoarthritis, BioResearch Open Access 6:1, 151-158, DOI: 10.1089/biores.2017.0027.

\begin{tabular}{|c|}
\hline $\begin{aligned} & \text { Abbreviations Used } \\
& \mathrm{AAI}=\text { autologous anti-inflammatory } \\
& \mathrm{ACD}-\mathrm{A}=\text { Anticoagulant Citrate Dextrose Solution-Formula } \mathrm{A} \\
& \mathrm{AE}=\text { adverse events } \\
& \mathrm{APS}=\text { Autologous Protein Solution } \\
& \mathrm{IDE}=\text { Investigational Device Exemption } \\
& \mathrm{IL}-1 \mathrm{ra}=\text { interleukin }-1 \text { receptor antagonist } \\
& \mathrm{K}-\mathrm{L}=\text { Kellgren-Lawrence } \\
& \mathrm{KOOS}=\text { Knee Injury and Osteoarthritis Outcome Score } \\
& \mathrm{NRS}=\text { Numeric Rating Scale } \\
& \mathrm{OA}=\text { Osteoarthritis } \\
& \text { OMERACT-OARSI }=\text { Outcome Measures in } \\
& \quad \text { Rheumatology-Osteoarthritis } \\
& \text { Research Society International } \\
& \mathrm{PBML}=\text { peripheral blood mononuclear cell leukocyte } \\
& \text { STNF- } \mathrm{R}=\text { soluble tumor necrosis factor receptors } \\
& \mathrm{WBC}=\text { white blood cell } \\
& \text { WOMAC }=\text { Western Ontario and McMaster Universities } \quad \text { Osteoarthritis Index }\end{aligned}$ \\
\hline
\end{tabular}

\section{Publish in BioResearch Open Access}

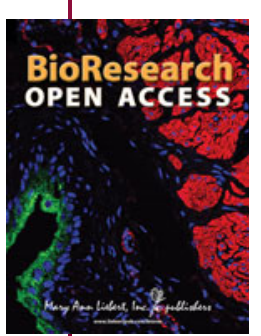

- Broad coverage of biomedical research - Immediate, unrestricted online access - Rigorous peer review - Compliance with open access mandates - Authors retain copyright

- Highly indexed

- Targeted email marketing 